TITLE:

\title{
Distinct neural firing mechanisms to tonal stimuli offset in the inferior colliculus of mice in vivo.
}

$\operatorname{AUTHOR}(S)$ :

Kasai, Masatoshi; Ono, Munenori; Ohmori, Harunori

\section{CITATION:}

Kasai, Masatoshi ...[et al]. Distinct neural firing mechanisms to tonal stimuli offset in the inferior colliculus of mice in vivo.. Neuroscience research 2012, 73(3): 224-237

ISSUE DATE:

2012-07

URL:

http://hdl.handle.net/2433/157930

\section{RIGHT:}

(C) 2012 Elsevier Ireland Ltd and the Japan Neuroscience Society.; この 論文は出版社版でありません。引用の際には出版社版をご確認ご利用 ください。; This is not the published version. Please cite only the published version. 


\title{
TITLE:
}

Distinct Neural Firing Mechanisms to Tonal Stimuli Offset in the Inferior Colliculus of Mice in vivo

\section{Authors:}

Masatoshi KASAI*1, Munenori ONO, Harunori OHMORI

\section{Affiliation:}

Department of Neurobiology, Kyoto University Graduate School of Medicine, Kyoto, 606-8501, Japan

Tel.: +81-75-753-4356

Fax: $+81-75-753-4349$

E-mail

MK: kassai@nbiol.med.kyoto-u.ac.jp, kassai@nips.ac.jp

MO: ono@nbiol.med.kyoto-u.ac.jp

HO: ohmori@nbiol.med.kyoto-u.ac.jp

\section{Corresponding author:}

*To whom correspondence should be addressed;

Masatoshi KASAI

\begin{abstract}
Offset neurons, which fire at the termination of sound, likely encode sound duration and serve to process temporal information. Offset neurons are found in most ascending auditory nuclei; however, the neural mechanisms that evoke offset responses are not well understood. In this study, we examined offset neural responses to tonal stimuli in the inferior colliculus (IC) in vivo with extracellular and intracellular recording techniques in mice. Based on peristimulus time histogram (PSTH) patterns, we classified extracellular offset responses into four types; Offset, Onset-Offset, Onset-Sustained-Offset and Inhibition-Offset types. Moreover, using in vivo whole-cell recording techniques, we found that offset responses were generated in most cells through the excitatory and inhibitory synaptic inputs. However, in a small number of cells, the offset responses were generated as
\end{abstract}

\footnotetext{
${ }^{1}$ Present Adress: Department of Developmental Physiology, National Institute for Physiological
} Sciences, Okazaki, Japan. 
a rebound to hyperpolarization during tonal stimulation. Many offset neurons fired robustly at a preferred duration of tonal stimulus, which corresponded with the timing of rich excitatory synaptic inputs. We concluded that most IC offset neurons encode the termination of the tone stimulus by responding to inherited ascending synaptic information, which is tuned to sound duration. The remainder generates offset spikes de novo through a postinhibitory rebound mechanism.

\section{Highlights:}

IC neurons respond to tonal duration and fire at the offset in four patterns.

Timing and balance between EPSC and IPSC determined the pattern of offset firings.

Some neurons fire from the rebound depolarization to tone induced inhibitory inputs.

Charge carried by offset synaptic inputs was largest at the preferred tonal duration.

\section{Keywords:}

Audition, Inferior Colliculus, Whole-cell Recording, Offset Response, Duration Tuning, Mice

\section{Acknowledgements:}

We appreciate Prof. Tom C.T. Yin (Department of Neuroscience, UW-Madison School of Medicine and Public Health) for reading the manuscript and providing helpful comments, Prof. Y. Yanagawa (Department of Genetic and Behavioral Neuroscience, Gunma University Graduate School of Medicine) for providing GAD67-GFP knock-in mice, and Prof. T. Kaneko and Dr. K. Nakamura (Department of Morphological Brain Science, Graduate School of Medicine, Kyoto University) for the generous gift of the antieGFP antibody. 


\section{Intoroduction}

Natural sounds have several features, such as frequency, level, duration and timing, and these features provide abundant information for animal communication. Inferior colliculus (IC) is believed to be the first integrative center for processing complex sounds because all sound information converges in this brain region. Synaptic inputs from the unilateral and bilateral lower auditory nuclei, as well as the descending projections from the auditory cortices, all converge in this region (reviewed in Casseday and Covey 1996). As a result, IC neurons show a variety of firing patterns that represent various types of auditory information (Ehret and Moffat 1985; Knudsen and Konishi 1978; Toronchuk et al. 1992; Casseday et al. 1994; Wang et al. 2006). Sound duration is thought to be one of the essential information for auditory processing and complex cognitive function. To represent sound duration, certain neurons fire continuously during the stimulus, and other neurons fire at the stimulus offset to encode its termination. The neurons that encode the termination of sound are called offset neurons. These neurons are found in the IC (bat: Casseday et al. 1994; Voytenko and Galazyuk 2008; rodent: Brand et al. 2000; Pérez-González et al. 2006; cat: Radionova 1988) as well as in other auditory nuclei (cochlear nucleus, Young and Brownell 1976; superior olivary nuclei, Covey et al. 1991; Grothe 1994; Kuwada and Batra 1999; Behrend et al. 2002; Kulesza et al. 2003; nuclei of lateral lemniscus, Covey and Casseday 1991; medial geniculate body, He 2001; auditory cortex, Recanzone 2000; Scholl et al. 2010). Recently, the offset firing mechanism in superior paraolivary nucleus (SPON) was proposed to be due to a disinhibition mechanism (Kulesza et al. 2007). Moreover, postinhibitory rebound firing facilitates the preciseness of the offset responses (Felix et al. 2011; Kopp-Scheinpflug et al. 2011). A convergence of post-inhibitory rebound and delayed excitatory inputs has been suggested to create offset firing in duration-sensitive neurons in the IC (Casseday et al. 1994; Mora and Kössl 2004; Faure et al. 2003). Computational models have also been proposed for mechanisms that create fine duration selectivity (Aubie et al. 2009). Recent in vivo whole-cell recordings in the IC demonstrated the presence of offset spikes in bats and mice (Xie et al. 2007; Tan and Borst 2007); however, detailed offset firing mechanisms have not been determined. In this paper, we conducted in vivo recordings from offset neurons in the IC extracellularly and intracellularly in mice, and we found that offset firing is generated by at least two distinct neural mechanisms; specifically, 
one is a rebound firing that is induced by membrane hyperpolarization during tone and the other is the firing that is induced through direct excitatory synaptic inputs at the tonal offset.

\section{Materials and methods}

\subsection{Animal preparations}

Experimental procedures conformed to guiding principles for the care and use of animals in the field of physiological sciences set by the Japanese Physiological Society. ICR mice (Shimizu; Kyoto, Japan) were used in most experiments, and in some cases, mice expressing green fluorescent protein (GFP) under the control of glutamate decarboxylase 67 (GAD67) were used in combination with juxtacellular staining to identify GABAergic neurons (GAD67-GFP knock-in mice; Yanagawa et al. 2001; Tamamaki et al. 2003). Animals of 4 to 12 weeks old were anesthetized with either chloral hydrate (intraperitoneal (i.p.) injection, $0.4 \mathrm{~g} / \mathrm{kg}$ ) or urethane (i.p. injection, $1.7 \mathrm{~g} / \mathrm{kg}$ ). Pinching the foot or tail almost every 30 minutes tested the anesthetic condition of the animal. When pinching reflex appeared, we added additional dose to maintain the level of anesthesia. We could not find particular difference in firing properties of IC offset neurons between these two anesthetics, in responses to tonal stimuli as well as in patterns of spontaneous firings; therefore we accumulated data from all offset neurons in the following analyses. The animals were mounted on a modified stereotaxic instrument (Narishige; Tokyo, Japan), and body temperature was maintained throughout the experiments using a warming pad. A plastic plate was fastened on the nasal bone to immobilize the head with dental cement (Fuji Ionomer Type II: GC; Tokyo, Japan), and a pair of hollow ear bars was attached to the opening of the ear canal. The unilateral surface of IC was exposed by removing a piece of the inter parietal bone and dura mater beneath it. Agarose gel (Agarose-LGT, Nacalai; Kyoto, Japan; $2-4 \%$ in a saline containing (in mM): $130 \mathrm{NaCl}, 4.5 \mathrm{KCl}, 2 \mathrm{CaCl}_{2}, 5$ PIPES$\mathrm{Na}(\mathrm{pH} 7.3)$ and $6 \%$ glucose) was placed on the exposed IC to prevent the surface from pulsating and drying.

\subsection{Auditory stimulation}

Tone stimuli were generated by a digital audio board (SE-200PCI, Onkyo; Tokyo, Japan) and were presented monaurally through the ear bar by a pair of tweeters (SRH291, Clarion; Saitama, Japan). The tones were in 30 steps of logarithmic-spaced frequencies that ranged from $1.7 \mathrm{kHz}$ to $72 \mathrm{kHz}$ at 10 different sound pressure levels (0-90 dBSPL in $10 \mathrm{~dB}$ 
increments). The sound pressure level (SPL) was calibrated using a standard microphone (type 4939: Bruel \& Kjaer) and spectrum of the tonal stimulus was measured before each experiment. Tone stimuli were 20 to $3000 \mathrm{~ms}$ in duration $(20,50,70,100,150,200,300$, 500, 1000, 2000 and $3000 \mathrm{~ms}$ ) with a 5-ms rise and decay and were shaped using a Hamming window. Tone stimuli and data acquisition were controlled using custom-made software in MATLAB (Math Works). All electrophysiological experiments were conducted in a soundproof booth (Onion Beto Booth-D50, Soundjapan; Saitama, Japan) placed within a sound attenuating room.

\subsection{Single unit recording}

Single unit activities were recorded in the unilateral IC by presenting pure tone stimuli monaurally to the contralateral ear. A thin-walled borosilicate glass capillary with a filament (GC150TF-10, Harvard Apparatus; MA, USA) was pulled (P-97, Sutter Instrument; CA, USA) and filled with a medium containing (in $\mathrm{mM}$ ); $150 \mathrm{NaCl}, 4.5 \mathrm{KCl}, 2$ $\mathrm{CaCl}_{2}, 1 \mathrm{MgCl}_{2}$ and 5 Hepes-KOH (pH 7.3). Biocytin (2\%, Sigma) was added to the pipette solution when juxtacellular staining was performed. The electrode was advanced vertically in 2- $\mu \mathrm{m}$ steps using a motor-driven micromanipulator (PC-5N, Narishige; Tokyo, Japan). The recording depth was 100-1000 $\mu \mathrm{m}$ below the surface of IC. Signals were amplified using an Axoclamp-2B (Molecular Devices; CA, USA). While advancing the electrode, the impedance was monitored by injecting negative current (200 pA for $50 \mathrm{~ms})$. When the impedance was increased, a tone stimulus of 70-90 dBSPL was presented. When single unit activities were observed, the auditory receptive field was first determined by presenting pure tone of 50-300 ms duration of the above 300 combinations of frequencies and SPLs. Each stimulus was presented in a pseudorandom order and repeated two or three times. The frequency to give the highest offset firing rate was then determined by applying isointensity tonal stimuli of 10-20 dB above the offset threshold SPL. Tones of this best responding frequency were applied to study the following offset firing property. The offset threshold SPL was determined as the smallest level that generated spikes at the stimulus offset. The extracted unit responses were confirmed as single unit activities based on the total superposing of offset spikes. Moreover, the onset, offset and spontaneous spike activities were superposed to the similar extent (data not shown). 


\subsection{PSTH, offset spikes, best duration and delay}

Peristimulus time histograms (PSTHs) of firing activity were calculated with a 1-ms bin width, in response to a tone of best responding frequency of 10-20 dBSPL above the offset threshold intensity, which was repeated more than 30 times at a fixed interval that was longer than $200 \mathrm{~ms}$. PSTHs were expressed as firing rates (spikes/s), which were calculated from the mean spike number at each bin divided by the bin width. Offset spikes were counted from PSTHs in a time window that was uniquely defined in each cell as the time range that covered the offset burst activity of the highest firing rate; the offset time window is indicated by two broken lines in Figures $2 \mathrm{~A}(\mathrm{c})-\mathrm{D}(\mathrm{c})$. When there were after-dischargelike activities, the offset spikes were counted from the first offset burst activity (indicated by the open arrowheads in Figures $4 \mathrm{~B}$ and $6 \mathrm{~A}$ ). In case spontaneous firings occurred, the time window covered the offset responses larger than two standard deviations (SDs) of the spontaneous firing rate (Figure 1D). Offset spike numbers within the time window were normalized by the largest offset spike number of the unit and were plotted as a function of tone duration. Tonal duration that generated the largest offset response was defined as the best duration. Delay of the offset spike was measured as the mean of the first offset spike time from stimulus termination. The SD of the offset spike time was adapted to measure the compactness of offset firings for the single unit and in vivo whole-cell recordings.

\subsection{Whole-cell recording}

Patch electrodes were used and had a resistance of 3-7 M $\Omega$ when filled with an internal medium containing (in $\mathrm{mM}$ ): $131 \mathrm{~K}$-gluconate, $4 \mathrm{KCl}, 3 \mathrm{MgCl}_{2}, 0.1$ EGTA, 5 $\mathrm{Na}_{2} \mathrm{ATP}, 0.3 \mathrm{Na}_{2} \mathrm{GTP}, 5 \mathrm{Na}_{2}$-phosphocreatine and 10 Hepes-KOH (pH 7.3). The electrode was inserted at the caudal part of IC to a depth of 100-500 $\mu \mathrm{m}$ from the surface. The electrode was advanced while applying a high positive pressure (7.8-9.8 $\mathrm{kPa}$ ) inside, while in the continuous single electrode voltage clamp (cSEVC) mode. A sudden increase in electrode resistance and oscillation of current occurred when the tip touched a cell (Margrie et al. 2002). Positive pressure was released and a slight negative pressure was applied to make a tight seal. After the tight seal was formed, the electrode potential was held at -60 $\mathrm{mV}$ while applying a modest negative pressure through a syringe. Further negative pressure achieved the whole-cell recording configuration. Resting membrane potential of offset neurons were $-66.1 \pm 5.7 \mathrm{mV}$ (Offset type, $\mathrm{n}=3$ ), $-55.0 \pm 10.0 \mathrm{mV}$ (Onset-Offset type, $\mathrm{n}=$ 
6, and -60.4 and $-42.2 \mathrm{mV}$ (Rebound type, $\mathrm{n}=2$ ). Auditory receptive fields and best responding frequency were determined under the current clamp as in the single unit recording. Series resistance was $93.9 \pm 40.5 \mathrm{M} \Omega(\mathrm{n}=11)$ and was canceled by a bridge balance circuit. Current clamp and cSEVC mode was adopted in the whole cell recordings.

In several figures (Figures 3A, 3D, 4A, 4B, 5A, 5B, 6A and 6D), the average voltage or current responses were overlaid by red on individual traces by black. Averaging was conducted after clipping action potentials or transient inward currents in individual traces to focus on subthreshold tonal responses.

Under the relatively high series resistance recording condition in the present experiments, holding the membrane potential at a depolarized destined potential was particularly difficult. We therefore compared the charge carried by the whole synaptic currents at a fixed membrane potential as a function of tonal duration in each neuron (Figure 5E). Electronic charges carried by synaptic currents were calculated by numerical integration (trapezoidal rule: trapz function in MATLAB) separately for the EPSC and IPSC after subtracting the mean baseline current of the immediate $50 \mathrm{~ms}$ before the tonal stimulus. Numerical integration was conducted on every trial over the period in which the current exceeded $30 \%$ of the peak synapse current level, which was performed to separate individual synaptic currents from the slow decay of the sustained onset or offset current. When synaptic responses repetitively emerged in groups (Figure 5B, arrowheads after stimuli), the first group of synaptic currents after the tonal offset was integrated (Figure 5E). Charge carried by the excitatory postsynaptic current (EPSC) was calculated when the membrane potential was $-75 \mathrm{mV}$ in every neurons (6 cells), near $\mathrm{E}_{\mathrm{Cl}}$. Charge carried by the inhibitory postsynaptic current (IPSC) was calculated by holding the membrane potential near $0 \mathrm{mV}$ ( 2 cells, Figure 5B). However, in several cases outward currents carried by the IPSC were integrated at -30 or $-40 \mathrm{mV}$ holding potentials ( 2 cells, see Figure $5 \mathrm{~A}$ ), because the current was unstable when the neuron was held near $0 \mathrm{mV}$ (see Figure 5A). These electrical charges were normalized separately for the EPSC and IPSC by the electrical charge carried at the tone of preferred duration, which was recorded at the same holding potential in each neuron (Figure 5E). In some experiments, the occurrence of the EPSC was visually detected from individual traces after low-pass filtering at $500 \mathrm{~Hz}$ and was rasterplotted (Figures 5C and D). 


\subsection{Recording and analyzing digital data}

Electrical responses were bandpass-filtered $(150 \mathrm{~Hz}$ to $10 \mathrm{kHz})$ and sampled at 50 $\mathrm{kHz}$ with 12-bit resolution in the whole-cell recordings and at 5 or $10 \mathrm{kHz}$ in the single unit recordings. The liquid junction potential, which was estimated to be $-12 \mathrm{mV}$, was corrected offline.

Offline analyses were performed using custom-written MATLAB software. The Rtest (version 2.9.2, http://www.r-project.org/) was used in some statistical analyses. Data are presented as the mean $\pm \mathrm{SD}$.

\subsection{Juxtacellular staining and histology}

Neurons were labeled juxtacellularly after unit recording (Pinault 1996) by applying anodal current pulses of ON (1-10 nA of $200 \mathrm{~ms})$ and OFF (200 ms) for up to $20 \mathrm{~min}$. Animals were sacrificed by a lethal dose of pentobarbiturate or urethane, and were fixed by transcardial perfusion using 4\% (w/v) formaldehyde and $0.01 \%(\mathrm{w} / \mathrm{v})$ glutaraldehyde in phosphate buffered saline (PBS at $\mathrm{pH}$ 7.4). After one day post-fixation, the brains were cryoprotected with $30 \%(\mathrm{w} / \mathrm{v})$ sucrose in PBS. Coronal brain slices were cut at a thickness of $40 \mu \mathrm{m}$ using a cryomicrotome (CM 30501, Leica; Berlin, Germany). Juxtacellular labeled neurons were visualized by incubation of the brain slices with Alexa 594-conjugated streptavidin (2 $\mu \mathrm{g} / \mathrm{ml}$, Invitrogen; CA, USA) in PBS containing 0.3\% (v/v) Triton X-100. GFP signals from GAD67-GFP knock-in mice were enhanced by affinity-purified rabbit antibodies to GFP (Tamamaki et al. 2000; Nakamura et al. 2008) and were visualized by Alexa 488-conjugated anti-rabbit IgG goat antibodies (Invitrogen).

\section{Results}

\subsection{Firing patterns of IC offset neurons using single unit recordings}

We recorded 610 neurons and found 56 neurons that responded at the offset of tone stimuli presented to the contralateral ear. We classified offset neurons into four types according to the pattern of PSTHs (Figures 1A-D). Figure 1A shows a unit that fired only at the tonal stimulus offset (Offset type; 23 out of 56 units). Figure 1B shows a neuron that fired at the onset and offset timings (17 out of 56 units; Onset-Offset type). In the 17 OnsetOffset-type units, we failed to change the tonal duration in one unit; however, 16 units were analyzed for duration sensitivity (Figures $1 \mathrm{~F}$ and J). Figure $1 \mathrm{C}$ shows a neuron that had a 
low sustained discharge during the tonal stimulus and fired at the onset and offset timings (9 out of 56 units, Onset-Sustained-Offset type). Two units in the Onset-Sustained-Offset type were atypical because they did not fire at the onset. Spontaneous firing was suppressed during the tonal stimulus in the unit illustrated in Figure 1D. The units that fired at the offset timing ( 7 out of 56 units) were classified as Inhibition-Offset type.

All offset neurons responded to various durations of stimuli (e.g., 20, 50, 100 and 200 ms, Figures 1A-D) and fired with a constant delay that was unique to each cell (mean offset delay ranged from 9.4 to $70.5 \mathrm{~ms}$ ). The delay of offset firings was not distinct in four PSTH types of unit responses. Furthermore, the offset firing rates varied depending on the duration of tone stimuli, and most neurons had a preferred tonal duration for firing, which was previously reported as the best duration (Pérez-González et al. 2006). Offset spike numbers were plotted against the tonal duration after normalization by the maximum offset spike number for each unit (Figures 1E-H). Black lines correspond to the units illustrated in Figures 1A-D, and gray lines show the other neurons of the corresponding PSTH patterns. The best duration is plotted in histogram form in Figures 1I-L. Most Offset-type, OnsetOffset-type and Onset-Sustained-Offset-type neurons preferred a tonal duration of 50-200 ms (Figures 1E, F, G, I, J and K). In contrast, the Inhibition-Offset-type neurons increased the firing rate relatively monotonously with the stimulus duration (Figures $1 \mathrm{H}$ and L). Spike numbers at the onset were hardly affected by the tone duration (Figures $1 \mathrm{~B}$ and $\mathrm{C}$ ).

\subsection{Receptive fields of the offset neurons}

Receptive fields are shown in Figure 2 for the four offset neurons presented in Figure 1. These neurons responded best to tones with a frequency of $11.9,7.9,6.2$ and $3.0 \mathrm{kHz}$ (Figures 2A(a)-D(a)). The best responding frequency for all the Offset-type neurons ranged from 2.4 to $21.1 \mathrm{kHz}(8.9 \pm 5.1 \mathrm{kHz}, \mathrm{n}=56)$. Most Offset neurons had a broad tuning property (half width; $1.9 \pm 1.0$ octave, $\mathrm{n}=56$ ), and the offset threshold level was high (66.4 $\pm 14.2 \mathrm{dBSPL}, \mathrm{n}=56$ ); therefore, the offset responses were recorded at relatively high tonal levels. Regardless of the high-level tonal stimuli, the firing responses were not distorted (Figures 2A(c)-D(c)). A unit shown in Figure 1A had the offset threshold of $70 \mathrm{dBSPL}$, and the offset response was induced by $80 \mathrm{dBSPL}$. Firing responses over the all stimulus frequency were accumulated and illustrated in the form of PSTHs (Figures 2A(c)-D(c)). Figure $2 \mathrm{~A}(\mathrm{c})$ indicates that the offset response property was not changed by the increase in 
the tonal level from 70 to $90 \mathrm{dBSPL}$. The Onset-Offset-type neuron shown in Figure 1B had a relatively low threshold for the offset neurons, and the onset spikes occurred near 30 dBSPL, while the offset response was detected at $40 \mathrm{dBSPL}$ (Figure 2B(a)-(c)). The tonal level did not affect the response pattern (Figure 2B(c)). Figure $2 \mathrm{C}$ shows the response of the unit in Figure 1C, and sustained responses emerged at approximately $40 \mathrm{dBSPL}$. The OnsetSustained-Offset response pattern was maintained at $90 \mathrm{dBSPL}$ (Figure 2C(c)). The Inhibition-Offset-type units had some spontaneous firing activity; therefore, determination of the tuning frequency was difficult, and inhibition was observed in a relatively broad frequency range (Figure 2D(b)). The offset response emerged at nearly $60 \mathrm{dBSPL}$ (between two vertical lines).

In each Onset-Offset-type and Onset-Sustained-Offset-type neuron, the tuning areas of the onset and offset responses nearly overlapped (Figures 2B(a)-(b) and 2C(a)-(b)).

\subsection{Post-inhibitory rebound firings from whole-cell recorded offset neurons}

Eleven offset neurons were detected in 69 whole-cell recordings of in vivo IC neurons, and two distinct offset firing mechanisms were found. The first type was rebound firing after a hyperpolarizing response to a tonal stimulus and offset spikes were generated without EPSC inputs (2 out of 11 neurons). Figure 3A shows representative current clamp traces (black, individual traces with spikes; red, averaged trace after clipping the spikes). Neurons generated immediate hyperpolarizing responses to tonal stimuli of various lengths (20, 50, 100 and $200 \mathrm{~ms}$ ) and fired after termination of the stimuli (100 and $200 \mathrm{~ms}$ ). Hyperpolarization occurred after a short delay from the tone onset $(50 \%$ rise time of IPSP was $28.6 \pm 5.6 \mathrm{~ms}$ for this neuron) and lasted for approximately $100 \mathrm{~ms}$ when we presented a short tone of 20 and $50 \mathrm{~ms}$ in duration (Figures 3A(a)-(b)). By a prolonged tonal stimulus of 100-200 ms, hyperpolarization was also prolonged and fired several spikes (Figures 3A and B) that were followed by depolarizing after potential (Figure 3A). The offset spikes occurred sparsely and the offset delay was dispersed; the SD of the offset delay was 41.9 ms. Several spikes occurred long after the termination of the tonal stimulus (200 ms, Figures $3 \mathrm{~A}(\mathrm{a})-(\mathrm{b}))$ and from depolarization preceding the tonal stimulus (Figure $3 \mathrm{~A}(\mathrm{~d})$ ), which we assumed to be a spontaneous activity. The number of offset spikes increased with the stimulus length (Figures 3B and C). Under the voltage clamp at $-60 \mathrm{mV}$, a sustained outward current that was probably attributable to the tone-induced IPSC, was recorded 
during the tonal stimulus; however, no obvious inward currents were detected after the termination of the tonal stimulus (Figure 3D). We used the pipette solution containing 10 $\mathrm{mM} \mathrm{Cl}{ }^{-}$with an estimated $\mathrm{E}_{\mathrm{Cl}}$ approximately $-75 \mathrm{mV}$ (extracellular $\mathrm{Cl}^{-}$concentration was assumed to be $150 \mathrm{mM}$; Zhou, et al. 2010; Scholl et al. 2010). A small outward current remained during the tonal stimulus, even when the neuron was voltage clamped nominally at $-90 \mathrm{mV}$ (Figure 3D, bottom trace). This could be because the actual membrane potential was more positive than we expected because of the limited space clamp conditions and a series resistance (discussed later). Furthermore, when we performed a current injection and hyperpolarized the membrane, rebound depolarization and spikes occurred (Figure 3E). Therefore, these offset spikes were probably generated de novo by rebound depolarization that followed membrane hyperpolarization during the tone stimulus. This type of offset firing mechanism may be responsible for the Inhibition-Offset-type unit activities because in both types, a strong inhibitory response was observed during tonal stimuli (Figure 1D). However, there are large differences in the distribution of offset spike timing (see Discussion).

\subsection{Timing and balance of excitatory and inhibitory synaptic inputs}

The majority of neurons fired in response to EPSPs after tonal stimulation (9 out of 11 units). However, there were large variations in the synaptic input patterns within this group of neurons. Most neurons received excitatory synaptic inputs at the stimulus onset and offset ( 8 out of 9 units, Figures 4 and 5), and one neuron received excitatory inputs only at the offset (1 out of 9 units, Figure 6).

Figure 4 shows two examples of whole-cell recorded neurons that responded to stimuli offsets without onset spike responses (Offset type, Figures 4A, C and E) and with onset spikes (Onset-Offset type, Figures 4B, D and F). The offset responses were most robust to tones that were $50 \mathrm{~ms}$ in duration in both cells (Figures $4 \mathrm{E}(\mathrm{b})$ and $\mathrm{F}(\mathrm{b})$ ), and the responses were smaller for shorter or longer tones. A short tone of $20 \mathrm{~ms}$ generated onset EPSPs that were subthreshold in both cells; however, an offset EPSP of long duration followed the onset EPSP in the Offset-type neuron (Figure 4A(a)) and a sharp offset EPSP with spikes followed in the Onset-Offset-type neuron (Figure 4B(a)). EPSPs after the tone stimulus had a longer duration in the Offset-type neurons than in the Onset-Offset neurons. The distribution of spike timings was relatively broad in the Offset type and was compact in 
the Onset-Offset type (Figures 4A-D); the SD of the offset spike time measured from the burst activity in Figure 4A and from the first burst activity (open arrowhead) in Figure 4B was $13.8 \mathrm{~ms}$ and $2.9 \mathrm{~ms}$ for the Offset- and Onset-Offset-type neurons, respectively. Afterdischarge-like spike activities followed in some neurons irrespective of the type of firing (see Figure 4B). The presence of after-discharge-like activities may reflect the slightly positive resting membrane potential that was measured preceding the tonal onset; -59.6 $\mathrm{mV}$ (Figure 4A) vs. $-55.3 \mathrm{mV}$ (Figure 4B). The slight hyperpolarization might have made the onset EPSP at a subthreshold level in the Offset neuron (Figure 4A). The spike observed at the stimulus onset in Figure 4A(d) was generated from depolarization preceding the tonal onset, and the two spikes in Figure 4A(a) were delayed so long after the tonal offset that they were probably generated spontaneously. Six of the eight neurons, including two sample neurons, did not fire in rebound to hyperpolarizing current injection; however, two other neurons fired in rebound to membrane hyperpolarization (data not shown).

Synaptic currents recorded in the Offset and Onset-Offset neurons of Figure 4 are illustrated in Figure 5A and B. Both neurons had EPSCs at the tonal onset followed by IPSCs when recorded at depolarized membrane potentials of $-40 \mathrm{mV}$ (Figure $5 \mathrm{~A}$ ) and 0 $\mathrm{mV}$ (Figure 5B). Outward currents that were recorded at $0 \mathrm{mV}$ at the tonal onset followed the timing of onset inward current recorded at $-75 \mathrm{mV}$ in the Onset-Offset neuron (Figure 5B). Moreover, repetitive peaks of EPSC (1st-3rd peaks were indicated as black arrowheads in Figure 5B) were precisely followed by IPSCs (Figure 5B, open arrowheads) in the OnsetOffset neuron; IPSCs delayed EPSCs by $7.3 \mathrm{~ms}$ (at the onset) and $8.9 \mathrm{~ms}$ (at the 1st offset) on average when measured at the individual peak. However, IPSCs were readily noticed only at the onset timing in the Offset neuron and were delayed $20.3 \mathrm{~ms}$ to the peak of the onset EPSCs (Figure 5A, asterisks). Raster plots of individual EPSCs are demonstrated in Figures 5C, D, for these neurons. Offset EPSCs occurred at dispersed timing in the Offset neuron (Figure 5C), while in the Onset-Offset neuron, the offset EPSCs occurred at relatively constant times and were cut out sharply (Figure 5D, black arrowheads). With the increase of tonal duration from $50 \mathrm{~ms}$ to $200 \mathrm{~ms}$, EPSCs occurred at more scattered timing in both neurons (Figures 5C (b) and D (b)); the SD of the occurrence time for offset EPSCs for the $50 \mathrm{~ms}$ and $200 \mathrm{~ms}$ tone was $19.3 \pm 20.7 \mathrm{~ms}$ and $28.8 \pm 26.2 \mathrm{~ms}$ in the Offset-type neurons $(\mathrm{p}=0.12)$, respectively, and $11.8 \pm 8.3 \mathrm{~ms}$ and $32.4 \pm 28.4 \mathrm{~ms}$ in Onset-Offset-type 
neurons $(\mathrm{p}<0.01)$, respectively. Prolongation of the tonal duration accompanied with a decrease of total electric charge carried by offset EPSCs; total electric charge decreased from $11.6 \pm 4.6$ to $5.4 \pm 1.0 \mathrm{pC}$ in the Offset-type neurons and from $6.3 \pm 1.4$ to $2.0 \pm 0.8$ $\mathrm{pC}$ in the Onset-Offset-type neurons at the $50 \mathrm{~ms}$ and $200 \mathrm{~ms}$ tones, respectively. These two tonal lengths corresponded to the best duration and a longer duration for the sample neurons (Figures 4E and F).

Total electric charge was calculated separately for the EPSCs and IPSCs in six neurons and was normalized to the corresponding electric charge at the best tonal duration (Figure 5E). Total electric charges carried by the EPSCs during stimulus were nearly independent of tonal duration (Figure 5E(a), black symbols), while electric charges carried by offset EPSCs were the largest at the best duration for the neuron (Figure 5E(b), black symbols). Electric charge carried by offset IPSCs decreased when the tone was longer than the best duration (Figure 5E(b), red symbols, $n=3$ ). Electric charge carried by onset IPSCs became larger with the tonal duration, which indicates that IPSCs continued during the tonal stimulation (Figure 5E(a), red symbols). In these evaluations, we normalized the measurements to those measured at the best tonal duration at the same membrane potential to facilitate comparison among neurons; EPSC at $-75 \mathrm{mV}$ for 6 neurons, and IPSC at $0 \mathrm{mV}$ (1 cells), $-30 \mathrm{mV}$ (1 cell), $-40 \mathrm{mV}(1$ cell).

One offset firing neuron did not receive excitatory synaptic inputs at the stimulus onset but received the inputs near the stimulus offset (Figure 6). The neuron demonstrated a compact offset burst firing (SD of $2.8 \mathrm{~ms}$, open arrowhead) and a pause, which was followed by a relatively long-lasting after-discharge-like firing activity (Figures 6A and B). We could only record the neuron at the membrane potential of $-60 \mathrm{mV}$, and the neuron did not show clear outward current that corresponded to the timing of the pause (Figure 6D); however, the pause cut off the burst activity and may reflect inhibitory inputs. This neuron preferred a tone duration of approximately $500 \mathrm{~ms}$ (Figure 6C) when the first offset activity was measured (open arrowheads in Figure 6A), and the neuron did not generate rebound spikes (Figure 6E). Absence of clear onset EPSCs indicates that this neuron could be a variation of Offset-type neurons (Figure 1A).

The balance and timing between excitatory and inhibitory synaptic inputs at the onset and offset of tonal stimulation may be responsible for the variety of Offset (Figure 1A) and 
Onset-Offset patterns (Figure 1B) of unit activity. We failed to whole-cell-record from cells that may explain the Onset-Sustained-Offset type mechanism (Figure 1C).

\subsection{Location and morphology of offset neurons}

Neurons in IC were so variable in morphology and physiology that it was difficult to search for neurons with specific properties during the experiments. By labeling the recorded neurons juxtacellularly, we attempted to understand the relationships among neuronal localization, cell morphology and tone-induced firing properties. Seven offset neurons were stained juxtacellularly, and one was further stained intracellularly by the whole-cell recording. Offset neurons had a tendency to be found in the caudal part of IC (Figure 7A) and were distributed from shallow to deep regions (Figure 7B). The recording depth had no particular correlation with the PSTH patterns (single units: $\mathrm{p}=0.40$; in vivo whole-cells: $\mathrm{p}=$ 0.16, Kruskal-Wallis test). Because whole-cell recordings from deep in vivo brain layers were difficult to obtain, the depth of the offset neurons may be biased to shallow regions in the dorsal cortex of IC (Figure 7B).

We traced and reconstructed the stained cells and identified three morphologically distinct cell types, as previously reported (Meininger et al. 1986; Oliver et al. 1991). The first type was a bipolar cell or a disc-shaped neuron (Figures 7C(a)-(c)). These neurons had two oblique dendrites that extended parallel to the tonotopic laminar direction. The second type was a multipolar or stellate neuron (Figure $7 \mathrm{C}(\mathrm{d})$ ), and the dendrites of these neurons diverged in the radial direction. The third neuron was a pyramidal-like cell (Figure 7C(e)). We could not find any correlation among the PSTH patterns, location or morphological features of these neurons. By using GAD67-GFP knock-in mice, we attempted to label offset cells juxtacellularly. Labeling succeeded in 19 IC neurons, and 7 of the neurons were offset neurons; however, no offset neurons were positive for the GFP signal (Figure 7D).

\section{Discussion}

We investigated neurons that responded to tonal offsets in the IC of mice in vivo and found a variety of unit response patterns in the generation of offset spikes. However, the extracellular recordings alone provide limited information on the basic mechanism of the firing activity, especially in understanding of the subthreshold responses. Therefore, we investigated the offset responses by the in vivo whole-cell recording technique. We found a 
small number of neurons generated spikes during rebound depolarization from hyperpolarization during tonal inputs, while most neurons generated offset spikes in response to excitatory synaptic inputs. The timing and balance between excitatory and inhibitory inputs varied and determined the firing features, including the compactness of offset firing activity. Most offset neurons showed preference for specific tone duration.

The number of offset neurons we could record using the whole-cell patch clamp represented $16 \%$ of the total whole-cell recordings (11 out of 69 cells); the percentage outnumbered the proportion of offset neurons found in the unit recording $(9.2 \%, 56$ out of 610 units). The larger proportion may be observed because we focused the recording in the caudal and shallower part of the IC (Figure 7A). Accordingly, some characteristics of the offset neurons may have been affected by these sampling biases. Moreover the small number of whole-cell recorded neurons in vivo may not be suitable to generalize the character of the IC offset neurons; however, we believe that our observations still indicate certain distinctions in the mechanism of the offset firing among IC neurons.

\subsection{Distinct mechanisms to generate offset responses in the IC}

Two distinct mechanisms have been suggested to explain the offset responses to sound; post-inhibitory rebound excitation (Casseday et al. 1994; Faure et al. 2003; Mora and Köss1 2004) and excitatory synaptic inputs at sound offset timing (Casseday et al. 1994; Tan and Borst 2007; Xie et al. 2007). The post-inhibitory rebound excitation is generated through the intrinsic membrane property that follows the tonal inhibition. Most neurons in the IC receive inhibitory innervations; thus the offset firing and sound duration sensitivity could be affected by inhibitory modulation (LeBeau, et al. 2001; Zheng and Knudsen 1999). However, the rebound-offset neurons have not been demonstrated in preceding studies of in vivo whole-cell recordings (Tan and Borst 2007; Xie e al. 2007). In this study we found, although small in numbers (2/11), that some offset neurons generated rebound spikes from hyperpolarization during a tone stimulus (Figure 3). Although voltage clamp recordings were not perfect in these experiments and the membrane potential was not ideally clamped, EPSC should be still observed near the resting potential of the cell; while the recording of IPSC was difficult because of the small driving force. Nevertheless, the tonal stimuli evoked the outward-going synaptic current but the inward-going synaptic current was not observed 
at the tonal offset, which might indicate an absence of excitatory synaptic inputs evoked by the tone of best responding frequency in these neurons (Figure 3).

In the most other offset neurons, EPSPs were found at the tonal offset and the offset firings were affected by the tonal duration (Figures $4 \mathrm{~A}$ and $\mathrm{B}$ ). Because of the relatively high series resistance in the present experiments and the consequent voltage-clamp error and low pass filtering effects, quantitative analyses were limited. Thus, we analyzed the effects of tonal duration on the total charge carried by synaptic inputs in reference to the charge carried by the preferred tonal duration in each cell. We found that the electrical charge carried by the offset EPSC was the largest at the preferred tonal duration (Figure 5Eb). This observation may indicate that the offset responses were attributed to the excitatory synaptic activities from, for example, the lower brainstem nuclei. Preceding studies in the bat demonstrated that duration sensitive neurons were found only in the IC and failed to show them in the lower nuclei (Casseday et al. 1988; Covey and Casseday 1991; Covey 1993; Casseday et al. 1994). Thus, some models have been proposed that duration sensitivities first emerge in the IC (Faure et al, 2003; Aubie et al, 2009). However, these models may not be consistent with our observation of excitatory synaptic inputs in the offset responses; particularly the duration sensitive excitatory synaptic inputs were not included in these models. Offset neurons have been reported in the other species in the lower brainstem nuclei such as the cochlear nucleus (cats: Shofner and Young 1985), the superior olivary complex (gerbils: Grothe 1994; Kuwada and Batra 1999; Behrend et al. 2002; rats: Kulesza et al. 2003) and lateral lemniscus (rats: Bajo et al. 1998; rabbits: Batra and Fitzpatrick 1999). Perhaps the most well understood offset firing mechanism in the auditory system is the one found in the superior paraolivary nucleus, SPON in mice. Neurons in the SPON generate offset spikes by disinhibition mechanism (Kadner et al. 2006; Kulesza et al. 2007; Kadner and Berrebi 2008), and the post-inhibitory rebound-firing mechanism enhanced the temporal preciseness of firings (Felix et al. 2011, Kopp-Scheinpflug et al. 2011). However, the SPON neurons make GABAergic projections to the IC (Kulesza and Berrebi 2000; González-Hernández et al. 1996; Saldaña et al. 2009) and are not appropriate as the direct source of offset excitatory inputs to the IC offset neurons. Some neurons in the cochlear nucleus $(\mathrm{CN})$ that generate Onset-Offset type response to sound could be another candidates; type IV neurons, which exhibit inhibitory responses to wide range of tone 
frequency (Shofner and Young 1985; Young and Brownell 1976; Ding et al. 1999). These neurons are in the deep layer of dorsal cochlear nucleus, DCN (Shofner and Young 1985), and Giant Cell is one of the major projection neurons in the deep layer of the DCN, which may make excitatory projections to the contralateral IC through the dorsal acoustic stria (Ryugo and Willard 1985; Osen 1972; Young 1980; Kane et al. 1981; Davis and Young 2000). Projection neurons of the DCN including Giant cells show onset-offset type firing activities; type IV responses (Young 1980). Furthermore, the axonal projection from the CN to IC, especially from the ventral part of the $\mathrm{DCN}$, has projections that terminate in the dorso-caudal region of the IC in the gerbil (Cant and Benson 2006; Cant and Benson 2008). Giant Cells in the DCN likely receive inputs of broad frequency range and has a relatively broadband frequency tuning (Ryugo and Willard 1985; Zhang and Oertel 1993; Kemmer and Vater 2001; Davis and Young 2000; Navawongse and Voigt 2009). These features of Giant Cells are compatible with the features of the IC offset neurons and could be a candidate for the source of ascending synaptic inputs (Figures 7A and 2A(a)-D(a); Chen 1998).

\subsection{Factors that affect temporal patterns of the offset responses}

The Onset-Offset neurons had the transient onset depolarization and spikes. The voltage clamp recording showed that the transient EPSC and IPSC underlay the onset depolarization (Figure 5). The IPSCs that occurred immediately after the EPSCs contributed to the compactness of spike timing (Figures $4 \mathrm{~B}$ and $5 \mathrm{~B}$ ) as indicated by previous study that was performed in the auditory cortex (Wehr and Zador 2003); while a prolonged offset spiking activities was observed in the case IPSCs did not follow EPSCs (Figures 4A and 5A). Accordingly, the timing and balance between EPSC and IPSC, as well as the resting potential of the neuron and the membrane excitability, could be factors to determine the temporal pattern of PSTH of the offset neurons. The balance of excitatory and inhibitory inputs may reflect neural circuits that project to the IC offset neurons, although little is known about the exact patterns of projection.

Contributions of the low-threshold T-type $\mathrm{Ca}^{2+}$ channels, $\mathrm{HCN}$ channels, or both are discussed for the generation of the rebound spikes (Li et al. 1998; Peruzzi et al. 2000; Sivaramakrishnan and Oliver 2001; Basta and Vater 2003; Koch and Grothe 2003; Wu et al. 2004; Ono et al. 2005; Felix et al. 2011, Kopp-Scheinpflug et al. 2011). The HCN channel 
was highlighted particularly for generating the sharp offset responses with short delays (Kopp-Scheinpflug et al 2011). However, the possibility of HCN channels seems low in our experiments, because we did not observe a clear depolarizing sag response during hyperpolarizing current injections (Figure 3E). Therefore, the T-type $\mathrm{Ca}^{2+}$ channels seemed more likely to generate the rebound spikes in the offset neurons we recorded.

Some units we classified as the Inhibition-Offset type likely generated spikes by the post-inhibitory rebound mechanism, because they demonstrated strong inhibition during tonal stimuli (Figures 1D). The Inhibition-Offset-type neurons in unit recordings and the rebound-offset neurons in whole-cell recordings may not have preferred tonal duration and most of them increased the firing activity with the prolongation of tonal stimulus (Figures $1 \mathrm{H}$ and $3 \mathrm{C}$ ). This nature was different from other offset neurons in the IC (Figure 1). The Inhibition-Offset neurons and the rebound-type offset neurons (Figures 1 and 3) exhibited some common properties but there were slight differences between them as follows. The unit responses of the Inhibition-Offset neurons were more compact with a shorter delay after the tonal offset while the whole-cell spike activities of rebound-type neurons were more dispersed after the tonal offset. This difference may be attributed to different recording methods, since the whole-cell recordings likely result in the rundown of some critical channels leading to the dispersed offset firings with longer delay (Figure 3).

\subsection{Information pathways for the sound duration}

Sensitivity to the sound duration is one of the essential capabilities of auditory function. Offset neurons in the IC are sensitive to tonal duration and believed to play important roles in echolocation or in speech communication (Casseday et al. 1994; Ehrlich et al. 1997; Qin et al. 2009; Bregman et al. 1994). Due to the distinct biological meanings of sound duration in different species, distinct neural mechanisms or neural network systems for encoding the sound offsets have developed during the evolution of the auditory system (Sayegh et al. 2011; Pérez-González et al. 2006; Casseday et al. 1988). Therefore, several offset neurons with a variety of features may cooperate to represent specific sound duration and sound features. Offset neurons in the medial geniculate body and auditory cortex are segregated functionally and spatially in ON and OFF information pathways (He 2001; Scholl et al. 2010). Accordingly, there could be specific offset information transmission pathways from the lower auditory nuclei to the auditory cortex, which process the 
termination or the duration of sound. We need further experiments to understand the neural basis of processing and encoding of sound duration in the IC and throughout the auditory system.

\section{GRANT}

This work was supported by a grant in aid from JSPS to HO (20220008).

\section{DISCLOSURES}

This manuscript has not been published and is not under consideration for publication elsewhere. All authors have read the manuscript and have approved submission. No conflicts of interest, financial or otherwise, are declared by the authors.

\section{FIGURE LEGENDS}

Figure 1, Response patterns and duration sensitivities of IC offset neurons to tone stimuli

PSTHs representing 4 types of offset firing activity. $A-D$, responses to 4 durations of tones at best responding frequency (20, 50, 100 and $200 \mathrm{~ms})$ in Onset, Onset-Offset, OnsetSustained-Offset and Inhibition-Offset type units (PSTH bin width was $1 \mathrm{~ms}$ ). Gray shades indicate the duration of the tonal stimulus in here and subsequent figures. $A$, this unit responded best at $11.9 \mathrm{kHz}$ and the offset threshold was $70 \mathrm{dBSPL}$, and the responses at 80 dBSPL are shown. The offset threshold, stimulus frequency and SPL of PSTHs for other neurons in this figure are as follows: $B, 40 \mathrm{dBSPL}$ at $7.9 \mathrm{kHz}, 40 \mathrm{dBSPL}$;, $40 \mathrm{dBSPL} 6.2$ $\mathrm{kHz}, 50 \mathrm{dBSPL}$; and $D, 60 \mathrm{dBSPL}, 3.0 \mathrm{kHz}, 70 \mathrm{dBSPL}$. Numbers at the top of each panel indicate the number of units of each firing type out of 56 offset units. Broken lines in $D$ indicate $2 \mathrm{SD}$ of the spontaneous activity. $E-H$, duration tuning properties of the offset neurons. Firing rates were normalized to the maximum firing rate of the units and are plotted against tonal duration. Black lines indicate neurons shown in $A-D$. All other neurons of corresponding PSTH types are shown in gray lines. $I-L$, histograms of the best duration; abscissa are presented in a logarithmic scale. 


\section{Figure 2, Frequency tuning patterns of IC offset neurons}

Frequency tuning and SPL dependence of the firing pattern of neurons presented in Figure 1. $A(a)-D(a)$; Frequency tuning of offset responses, which were counted after the termination of tonal stimuli. $A(b)-D(b)$; frequency tuning properties obtained from responses during tonal stimulation. The firing did not occur in $A(b)$, occurred at the onset in $B(b)$, and throughout the tonal stimulus in $C(b)$ while the firing was inhibited during the tone in $D(b)$. The broken line in $D(b)$ indicates the border of the inhibitory tuning area. Color code (on the right of Figure $A(b))$ shows the average number of spikes per stimulus at the corresponding SPL and frequency for Figure $A(a)-D(b)$. $A(c)-D(c)$; Spike responses were accumulated over the stimulus frequency $(1.7-72 \mathrm{kHz}$ ) in the form of PSTH (bin width: $4 \mathrm{~ms}$ ). The firing pattern of the offset neurons was not affected by the tonal stimulus intensity. Red bars on the abscissa indicate tonal stimulus. Two vertical broken lines indicate the time window to measure the offset responses of each neuron in Figure 1. Color codes show the average number of spikes for the 50-ms tonal duration.

\section{Figure 3, Whole-cell recordings from the post-inhibitory rebound type offset neuron}

Whole-cell responses of rebound-type neurons. A, Tonal responses of membrane potential and spike activities to tone stimuli of best responding frequency $(3.6 \mathrm{kHz})$ of 50 dBSPL for $20 \mathrm{~ms}$ (a) $50 \mathrm{~ms}$ (b) $100 \mathrm{~ms}$ (c) and $200 \mathrm{~ms}$ (d). Traces in black indicate 20 sequentially recorded traces. Traces in red indicate the average after action potentials were truncated. $B$, PSTHs calculated from a repetition of 30-39 tonal stimuli for the responses corresponding to A. $C$, the duration tuning of this cell (black) and another rebound-type cell (gray). $D$, synaptic currents induced by tonal stimuli recorded at $-60 \mathrm{mV}$ (top) and $-90 \mathrm{mV}$ (bottom) under voltage clamp. Black traces indicate individual trials and red indicates averages. $E$, voltage responses to current injections of $500 \mathrm{~ms}$. In this figure, black indicates a response to positive current, red indicates negative current and gray indicates no current injection. At the bottom, the current injection pattern is illustrated. 
Figure 4, Responses from two types of whole-cell recorded offset neurons to tone stimuli

Two examples of offset firing neurons. $A$ and $B$, one neuron fired only at the offset timing $(A$, Offset) and another fired at the onset and offset times ( $B$, Onset-Offset). Tonal duration was from 20 to $200 \mathrm{~ms}$, and the best responding frequency were $5.1 \mathrm{kHz}(A)$ and $14.4 \mathrm{kHz}(B)$. $C$ and $D$, PSTHs (bin width, $1 \mathrm{~ms}$ ) of neurons in $A$ and $B$, respectively. Tonal stimulus was repeated $70(a), 110(b), 100(c)$ and 30 times $(d)$ for A, and $31(a), 58(b), 73$ $(c)$ and 30 times $(d)$ for B. $E$ and $F$, duration tuning properties. Black lines indicate neurons shown in $A$ and $B$. All other neurons of corresponding PSTH patterns are shown in gray lines.

\section{Figure 5. Modulation of offset EPSCs by tonal duration}

Current responses to tonal stimuli of Offset and Onset-Offset neurons. $A$ and $B$, synaptic currents from neurons shown in Figure $4 A$ (Offset) and $B$ (Onset-Offset), respectively. Tonal stimuli of $50 \mathrm{~ms}(a)$ and $200 \mathrm{~ms}(b)$ were applied, and synaptic current responses were recorded at two membrane potentials, as indicated in each panel. Traces in black indicate individual current responses, and red indicate the averaged responses. Asterisks in $A$ indicate outward current peaks, and open and filled arrowheads in $B$ indicate peaks of ensemble averaged IPSCs and EPSCs, respectively. Note that the EPSC in $B$ was closely followed by the IPSC. $C$ and $D$, individual EPSCs were detected in each current trace (black traces in $A$ and $B)$ and were plotted in raster form $((a), 50 \mathrm{~ms} ;(b), 200 \mathrm{~ms}$ tone; shaded region). Filled arrowheads indicate the timing of peaks of ensemble averaged offset EPSCs. E, electrical charges carried by EPSCs (black) and IPSCs (red) were normalized to the charge carried at the best duration and were plotted against the tonal duration difference from the best duration for six neurons for the onset and sustained response during stimulus (a) and after termination of the stimuli (b). Different symbols indicate different cells (Offsettype: filled symbols; Onset-Offset-type: open symbols). The abscissa indicates the difference of tonal duration from the best duration. 


\section{Figure 6, Offset neurons that received excitatory inputs only at the tonal offset}

Offset neurons that received only excitatory inputs. $A$ : firing responses to tone of best responding frequency $(10.8 \mathrm{kHz})$ for $400 \mathrm{~ms}(a)$ and $500 \mathrm{~ms}(b)$ duration. $B$, PSTHs obtained from current clamp recordings of 40-114 stimuli of the tone at best responding frequency that were $200-500 \mathrm{~ms}$ in duration. $C$, duration tuning property. $D$, synaptic current responses to a 500-ms tone at a holding potential of $-60 \mathrm{mV}$. E, firing responses (top) to current injections (bottom).

\section{Figure 7, Location and morphologies of IC offset neurons}

Recording locations and morphology of IC offset neurons. $A$, distribution of offset neurons in IC by juxtacellular labeling. $B$, recording depths of offset neurons; unit recordings (open circles: Offset; open triangles: Onset-Offset; open squares: OnsetSustained-Offset; crosses: Inhibition-Offset-type) and whole-cell recordings (filled circles: Offset; filled triangle: Onset-Offset-type; gray circles: rebound type). $C$, five examples of juxtacellular stained neurons; $(a)-(c)$ bipolar cells, $(d)$ multipolar cells and $(e)$ pyramidallike cells. $D$, fluorescent image of the cell illustrated in $C(c)$ filled with biocytin and visualized by Alexa594-conjugated streptavidin (red). Green fluorescence indicates GAD67-GFP enhanced by the anti-GFP antibody.

\section{REFERENCES}

Aubie, B., Becker, S., Faure, P.A., 2009. Computational models of millisecond level duration tuning in neural circuits. J Neurosci 29, 9255-9270.

Bajo, V.M., Villa, A.E., de Ribaupierre, F., Rouiller, E.M., 1998. Discharge properties of single neurons in the dorsal nucleus of the lateral lemniscus of the rat. Brain Res Bull 47, 595-610.

Basta, D., Vater, M., 2003. Membrane-based gating mechanism for auditory information in the mouse inferior colliculus. Brain Res 968, 171-178.

Batra, R., Fitzpatrick, D.C., 1999. Discharge patterns of neurons in the ventral nucleus of the lateral lemniscus of the unanesthetized rabbit. J Neurophysiol 82, 1097-1113.

Behrend, O., Brand, A., Kapfer, C., Grothe, B., 2002. Auditory response properties in the superior paraolivary nucleus of the gerbil. J Neurophysiol 87, 2915-2928. 
Brand, A., Urban, R., Grothe, B., 2000. Duration tuning in the mouse auditory midbrain. J Neurophysiol 84, 1790-1799.

Bregman, A.S., Ahad, P.A., Kim, J., 1994. Resetting the pitch-analysis system. 2. Role of sudden onsets and offsets in the perception of individual components in a cluster of overlapping tones. J Acoust Soc Am 96, 2694-2703.

Cant, N.B., Benson, C.G., 2006. Organization of the inferior colliculus of the gerbil (Meriones unguiculatus): differences in distribution of projections from the cochlear nuclei and the superior olivary complex. J Comp Neurol 495, 511-528.

Cant, N.B., Benson, C.G., 2008. Organization of the inferior colliculus of the gerbil (Meriones unguiculatus): projections from the cochlear nucleus. Neuroscience 154, 206-217.

Casseday, J.H., Covey, E., 1996. A neuroethological theory of the operation of the inferior colliculus. Brain Behav Evol 47, 311-336.

Casseday, J.H., Covey, E., Vater, M., 1988. Connections of the superior olivary complex in the rufous horseshoe bat Rhinolophus rouxi. J Comp Neurol 278, 313-329.

Casseday, J.H., Ehrlich, D., Covey, E., 1994. Neural tuning for sound duration: role of inhibitory mechanisms in the inferior colliculus. Science $264,847-850$.

Chen, G.D., 1998. Effects of stimulus duration on responses of neurons in the chinchilla inferior colliculus. Hear Res 122, 142-150.

Covey, E., 1993. Response properties of single units in the dorsal nucleus of the lateral lemniscus and paralemniscal zone of an echolocating bat. J Neurophysiol 69, 842-859.

Covey, E., Casseday, J.H., 1991. The monaural nuclei of the lateral lemniscus in an echolocating bat: parallel pathways for analyzing temporal features of sound. J Neurosci $11,3456-3470$.

Covey, E., Vater, M., Casseday, J.H., 1991. Binaural properties of single units in the superior olivary complex of the mustached bat. J Neurophysiol 66, 1080-1094.

Davis, K.A., Young, E.D., 2000. Pharmacological evidence of inhibitory and disinhibitory neuronal circuits in dorsal cochlear nucleus. J Neurophysiol 83, 926-940.

Ding, J., Benson, T.E., Voigt, H.F., 1999. Acoustic and current-pulse responses of identified neurons in the dorsal cochlear nucleus of unanesthetized, decerebrate gerbils. $\mathrm{J}$ Neurophysiol 82, 3434-3457. 
Ehret, G., Moffat, A.J.M., 1985. Inferior colliculus of the house mouse II. Single unit responses to tones, noise and tone-noise combinations as a function of sound intensity. J Comp Physiol A 156, 619-635.

Ehrlich, D., Casseday, J.H., Covey, E., 1997. Neural tuning to sound duration in the inferior colliculus of the big brown bat, Eptesicus fuscus. J Neurophysiol 77, 2360-2372.

Faure, P.A., Fremouw, T., Casseday, J.H., Covey, E., 2003. Temporal masking reveals properties of sound-evoked inhibition in duration-tuned neurons of the inferior colliculus. J Neurosci 23, 3052-3065.

Felix, R.A., Fridberger, A., Leijon, S., Berrebi, A.S., Magnusson, A.K., 2011. Sound rhythms are encoded by postinhibitory rebound spiking in the superior paraolivary nucleus. J Neurosci 31, 12566-12578.

González-Hernández, T., Mantolán-Sarmiento, B., González-González, B., Pérez-González, H., 1996. Sources of GABAergic input to the inferior colliculus of the rat. J Comp Neurol 372, 309-326.

Grothe, B., 1994. Interaction of excitation and inhibition in processing of pure tone and amplitude-modulated stimuli in the medial superior olive of the mustached bat. J Neurophysiol 71, 706-721.

He, J., 2001. On and off pathways segregated at the auditory thalamus of the guinea pig. J Neurosci 21, 8672-8679.

Kadner, A., Berrebi, A.S., 2008. Encoding of temporal features of auditory stimuli in the medial nucleus of the trapezoid body and superior paraolivary nucleus of the rat. Neuroscience 151, 868-887.

Kadner, A., Kulesza, R.J., Berrebi, A.S., 2006. Neurons in the medial nucleus of the trapezoid body and superior paraolivary nucleus of the rat may play a role in sound duration coding. J Neurophysiol 95, 1499-1508.

Kane, E.S., Puglisi, S.G., Gordon, B.S., 1981. Neuronal types in the deep dorsal cochlear nucleus of the cat: I. Giant neurons. J Comp Neurol 198, 483-513.

Kemmer, M., Vater, M., 2001. Functional organization of the dorsal cochlear nucleus of the horseshoe bat (Rhinolophus rouxi) studied by GABA and glycine immunocytochemistry and electron microscopy. Anat Embryol 203, 429-447. 
Knudsen, E.I., Konishi, M., 1978. A neural map of auditory space in the owl. Science 200, 795-797.

Koch, U., Grothe, B., 2003. Hyperpolarization-activated current (Ih) in the inferior colliculus: distribution and contribution to temporal processing. J Neurophysiol 90 , 3679-3687.

Kopp-Scheinpflug, C., Tozer, A.J.B., Robinson, S.W., Tempel, B.L., Hennig, M.H., Forsythe, I.D., 2011. The sound of silence: ionic mechanisms encoding sound termination. Neuron 71, 911-925.

Kulesza, R.J., Berrebi, A.S., 2000. Superior paraolivary nucleus of the rat is a GABAergic nucleus. J Assoc Res Otolaryngol 1, 255-269.

Kulesza, R.J., Kadner, A., Berrebi, A.S., 2007. Distinct roles for glycine and GABA in shaping the response properties of neurons in the superior paraolivary nucleus of the rat. J Neurophysiol 97, 1610-1620.

Kulesza, R.J., Spirou, G.A., Berrebi, A.S., 2003. Physiological response properties of neurons in the superior paraolivary nucleus of the rat. J Neurophysiol 89, 2299-2312.

Kuwada, S., Batra, R., 1999. Coding of sound envelopes by inhibitory rebound in neurons of the superior olivary complex in the unanesthetized rabbit. J Neurosci 19, 2273-2287.

LeBeau, F.E., Malmierca, M.S., Rees, A., 2001. Iontophoresis in vivo demonstrates a key role for $\mathrm{GABA}(\mathrm{A})$ and glycinergic inhibition in shaping frequency response areas in the inferior colliculus of guinea pig. J Neurosci 21, 7303-7312.

Li, Y., Evans, M.S., Faingold, C.L., 1998. In vitro electrophysiology of neurons in subnuclei of rat inferior colliculus. Hear Res 121, 1-10.

Margrie, T.W., Brecht, M., Sakmann, B., 2002. In vivo, low-resistance, whole-cell recordings from neurons in the anaesthetized and awake mammalian brain. Pflugers Arch 444, 491-498.

Meininger, V., Pol, D., Derer, P., 1986. The inferior colliculus of the mouse. A Nissl and Golgi study. Neuroscience 17, 1159-1179.

Mora, E.C., Kössl, M., 2004. Ambiguities in sound-duration selectivity by neurons in the inferior colliculus of the bat Molossus molossus from Cuba. J Neurophysiol 91, 22152226. 
Nakamura, K.C., Kameda, H., Koshimizu, Y., Yanagawa, Y., Kaneko, T., 2008. Production and histological application of affinity-purified antibodies to heat-denatured green fluorescent protein. J Histochem Cytochem 56, 647-657.

Navawongse, R., Voigt, H.F., 2009. Single neuron recordings in dorsal cochlear nucleus (DCN) of awake gerbil. Hear Res 255, 44-57.

Oliver, D.L., Kuwada, S., Yin, T.C., Haberly, L.B., Henkel, C.K., 1991. Dendritic and axonal morphology of HRP-injected neurons in the inferior colliculus of the cat. J Comp Neurol 303, 75-100.

Ono, M., Yanagawa, Y., Koyano, K., 2005. GABAergic neurons in inferior colliculus of the GAD67-GFP knock-in mouse: electrophysiological and morphological properties. Neurosci Res 51, 475-492.

Osen, K.K., 1972. Projection of the cochlear nuclei on the inferior colliculus in the cat. J Comp Neurol 144, 355-372.

Peruzzi, D., Sivaramakrishnan, S., Oliver, D.L., 2000. Identification of cell types in brain slices of the inferior colliculus. Neuroscience 101, 403-416.

Pérez-González, D., Malmierca, M.S., Moore, J.M., Hernández, O., Covey, E., 2006. Duration selective neurons in the inferior colliculus of the rat: topographic distribution and relation of duration sensitivity to other response properties. J Neurophysiol 95, 823836.

Pinault, D., 1996. A novel single-cell staining procedure performed in vivo under electrophysiological control: morpho-functional features of juxtacellularly labeled thalamic cells and other central neurons with biocytin or Neurobiotin. J Neurosci Methods 65, 113-136.

Qin, L., Liu, Y., Wang, J., Li, S., Sato, Y., 2009. Neural and behavioral discrimination of sound duration by cats. J Neurosci 29, 15650-15659.

Radionova, E.A., 1988. Off-responses in the auditory system in relation to the signal end phase and neuronal characteristic frequency. Hear Res 35, 229-235.

Recanzone, G.H., 2000. Response profiles of auditory cortical neurons to tones and noise in behaving macaque monkeys. Hear Res 150, 104-118. 
Ryugo, D.K., Willard, F.H., 1985. The dorsal cochlear nucleus of the mouse: a light microscopic analysis of neurons that project to the inferior colliculus. J Comp Neurol $242,381-396$.

Saldaña, E., Aparicio, M.-A., Fuentes-Santamaría, V., Berrebi, A.S., 2009. Connections of the superior paraolivary nucleus of the rat: projections to the inferior colliculus. Neuroscience 163, 372-387.

Sayegh, R., Aubie, B., Faure, P.A., 2011. Duration tuning in the auditory midbrain of echolocating and non-echolocating vertebrates. J Comp Physiol A Neuroethol Sens Neural Behav Physiol 197, 571-583.

Scholl, B., Gao, X., Wehr, M., 2010. Nonoverlapping Sets of Synapses Drive On Responses and Off Responses in Auditory Cortex. Neuron 65, 412-421.

Shofner, W.P., Young, E.D., 1985. Excitatory/inhibitory response types in the cochlear nucleus: relationships to discharge patterns and responses to electrical stimulation of the auditory nerve. J Neurophysiol 54, 917-939.

Sivaramakrishnan, S., Oliver, D.L., 2001. Distinct K currents result in physiologically distinct cell types in the inferior colliculus of the rat. J Neurosci 21, 2861-2877.

Tamamaki, N., Nakamura, K., Furuta, T., Asamoto, K., Kaneko, T., 2000. Neurons in Golgistain-like images revealed by GFP-adenovirus infection in vivo. Neurosci Res 38, 231236.

Tamamaki, N., Yanagawa, Y., Tomioka, R., Miyazaki, J.-I., Obata, K., Kaneko, T., 2003. Green fluorescent protein expression and colocalization with calretinin, parvalbumin, and somatostatin in the GAD67-GFP knock-in mouse. J Comp Neurol 467, 60-79.

Tan, M.L., Borst, J.G.G., 2007. Comparison of responses of neurons in the mouse inferior colliculus to current injections, tones of different durations, and sinusoidal amplitudemodulated tones. J Neurophysiol 98, 454-466.

Toronchuk, J.M., Stumpf, E., Cynader, M.S., 1992. Auditory cortex neurons sensitive to correlates of auditory motion: underlying mechanisms. Exp Brain Res 88, 169-180. Voytenko, S.V., Galazyuk, A.V., 2008. Timing of sound-evoked potentials and spike responses in the inferior colliculus of awake bats. Neuroscience 155, 923-936.

Wang, J., van Wijhe, R., Chen, Z., Yin, S., 2006. Is duration tuning a transient process in the inferior colliculus of guinea pigs? Brain Res 1114, 63-74. 
Wehr, M., Zador, A.M., 2003. Balanced inhibition underlies tuning and sharpens spike timing in auditory cortex. Nature 426, 442-446.

Wu, S.H., Ma, C.L., Kelly, J.B., 2004. Contribution of AMPA, NMDA, and GABA(A) receptors to temporal pattern of postsynaptic responses in the inferior colliculus of the rat. J Neurosci 24, 4625-4634.

Xie, R., Gittelman, J.X., Pollak, G.D., 2007. Rethinking tuning: in vivo whole-cell recordings of the inferior colliculus in awake bats. J Neurosci 27, 9469-9481.

Yanagawa, Y., Kaneko, K., Kanbara, N., Totsuka, M., Yagi, T., Obata, K., 2001. Development of mouse expressing GFP in GABAergic neurons. Neurosci. Res. Suppl. $25, \mathrm{~S} 77$.

Young, E.D., 1980. Identification of response properties of ascending axons from dorsal cochlear nucleus. Brain Res 200, 23-37.

Young, E.D., Brownell, W.E., 1976. Responses to tones and noise of single cells in dorsal cochlear nucleus of unanesthetized cats. J Neurophysiol 39, 282-300.

Zhang, S., Oertel, D., 1993. Giant cells of the dorsal cochlear nucleus of mice: intracellular recordings in slices. J Neurophysiol 69, 1398-1408.

Zheng, W., Knudsen, E.I., 1999. Functional selection of adaptive auditory space map by GABAA-mediated inhibition. Science 284, 962-965.

Zhou, Y., Liu, B.-H., Wu, G.K., Kim, Y.-J., Xiao, Z., Tao, H.W., Zhang, L.I., 2010. Preceding inhibition silences layer 6 neurons in auditory cortex. Neuron 65, 706-717. 
Figure 1
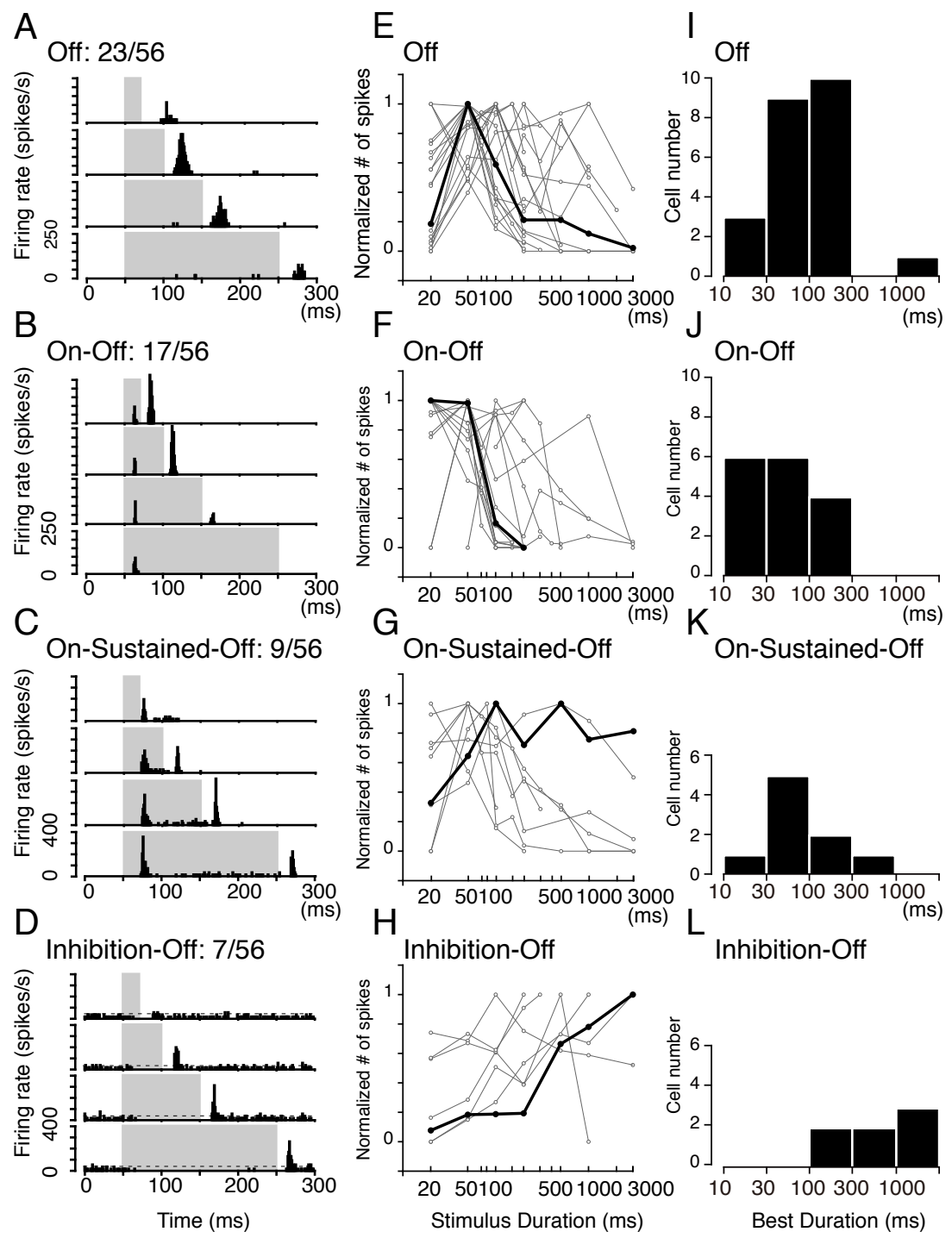

Inhibition-Off

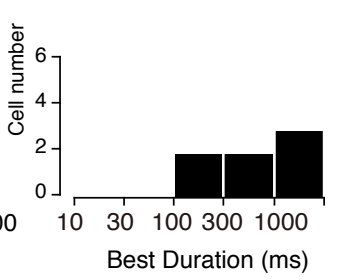


Figure 2

A: Offset

\section{(a)}

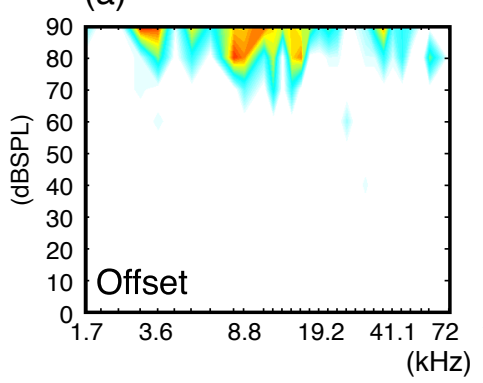

B: Onset-Offset

(a)

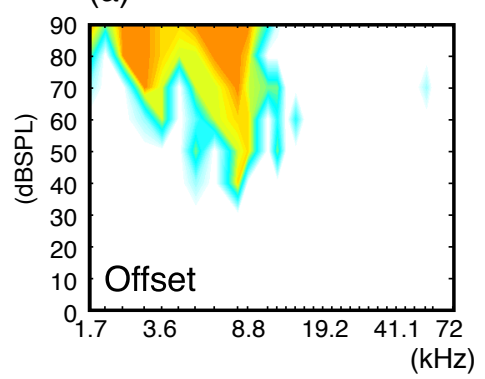

C: Onset-Sustained-Offset

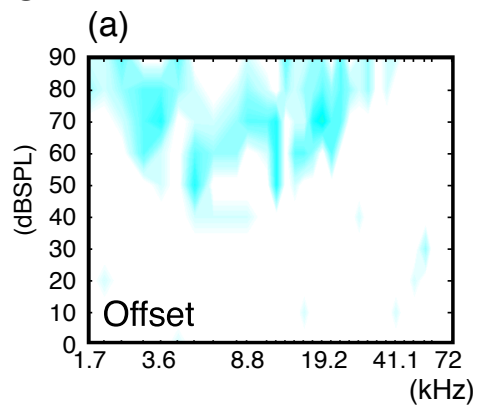

(b)

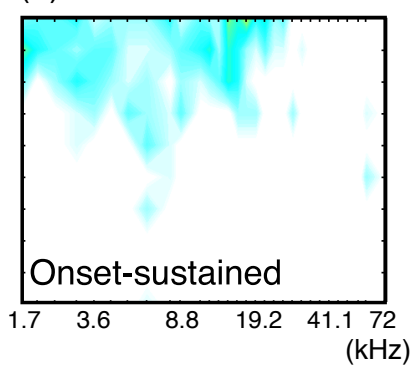

D: Inhibition-Offset

(a)

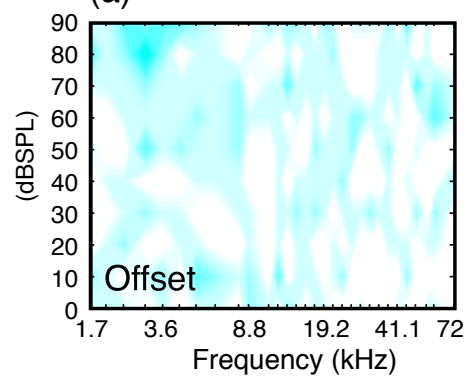

(b)

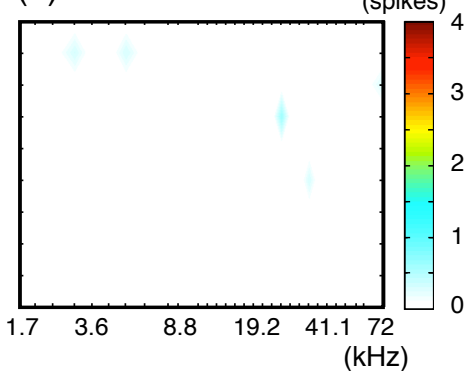

(b)

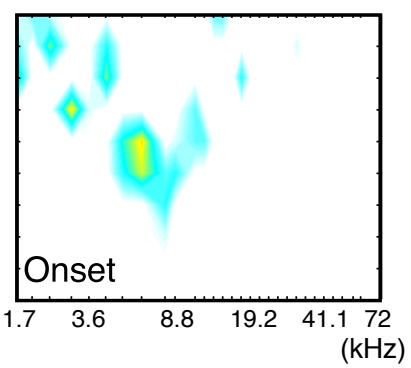

$(\mathrm{kHz})$ (b)

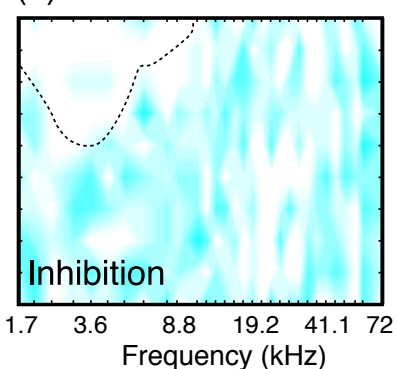

(c)

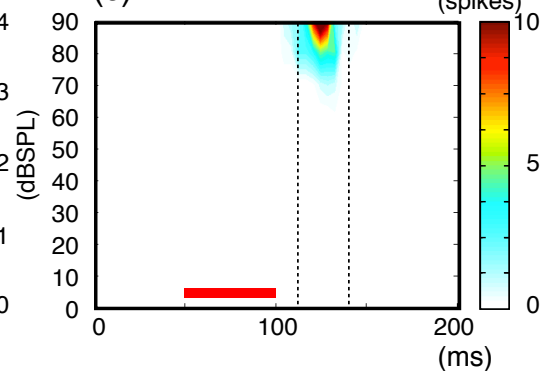

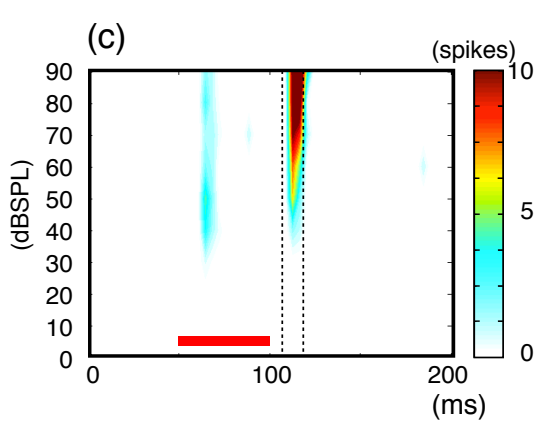
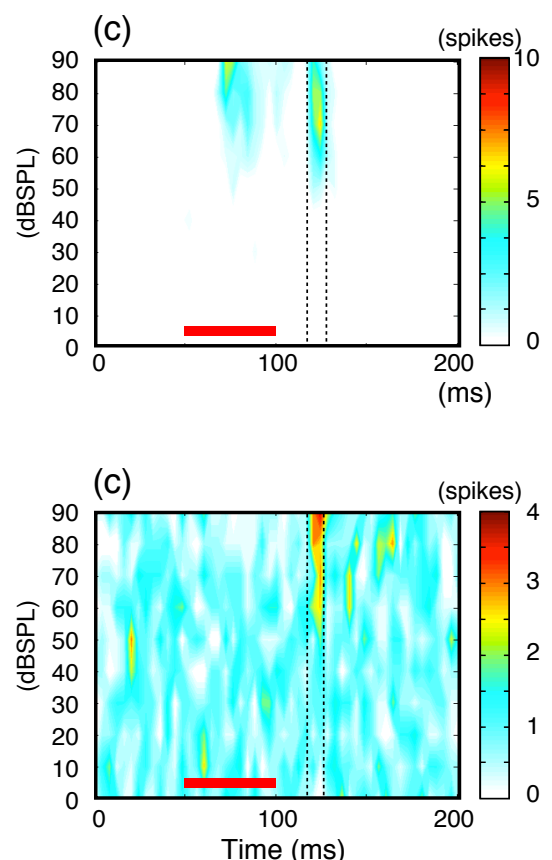
Figure 3

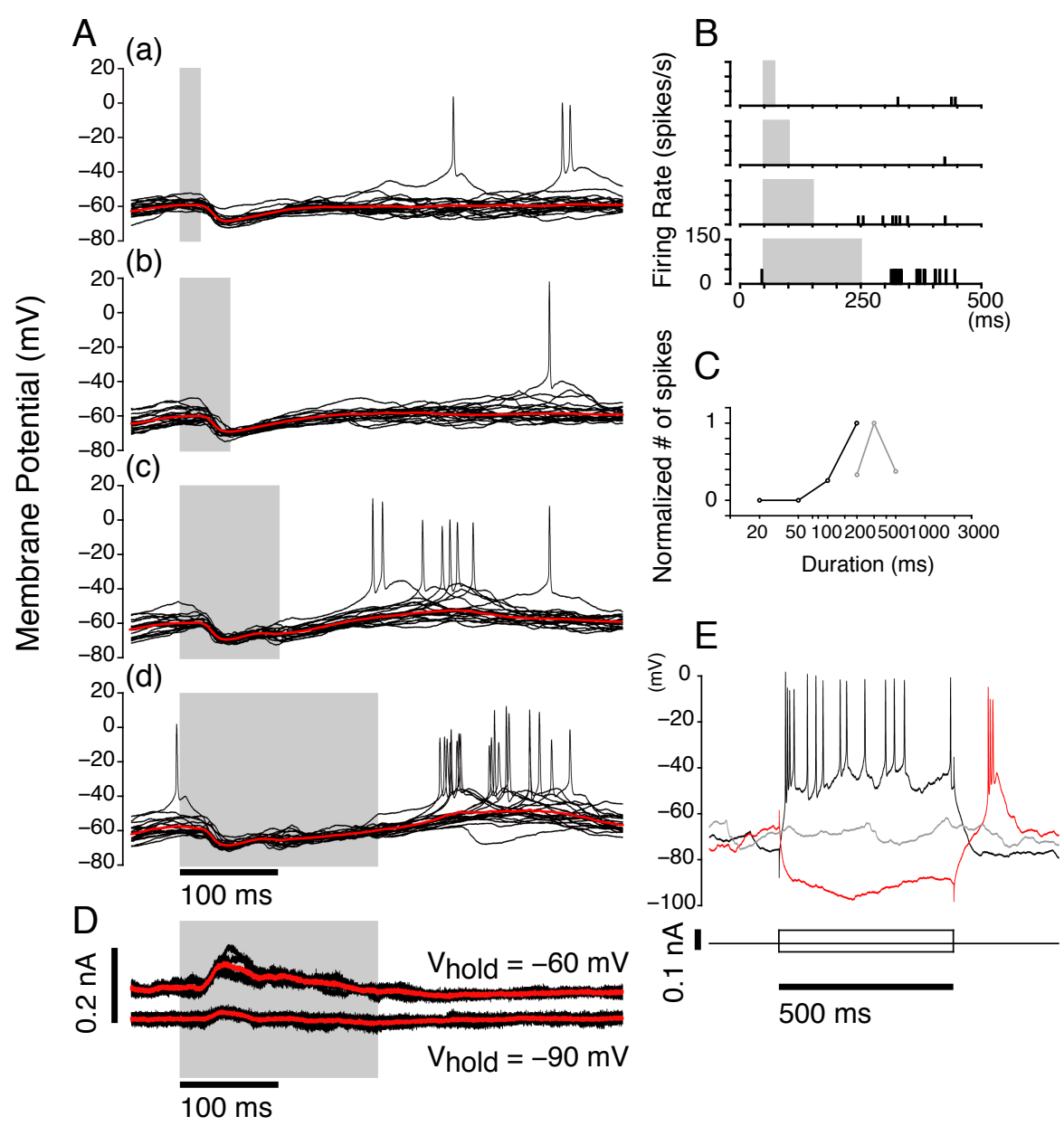


Figure 4

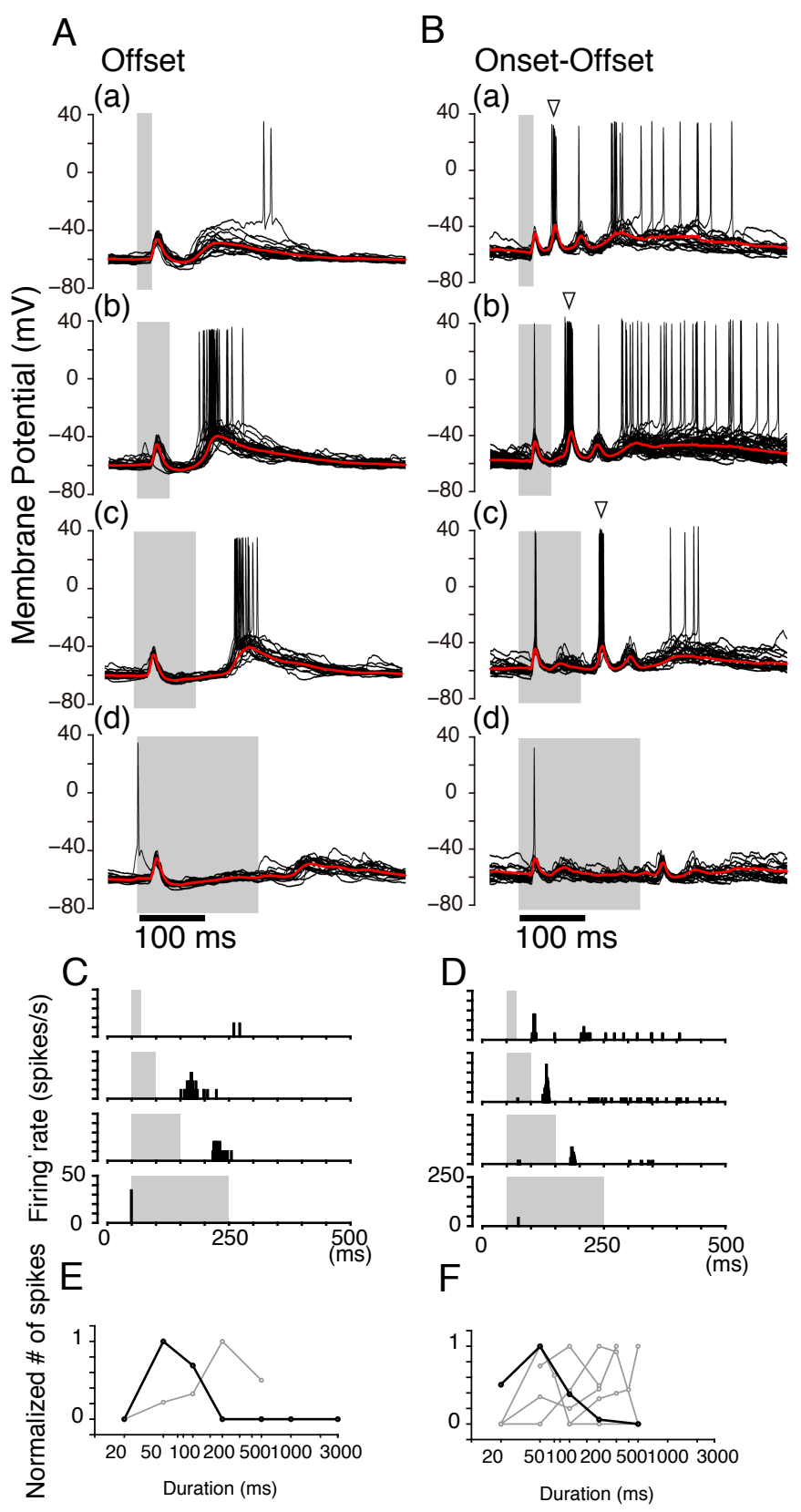


Figure 5

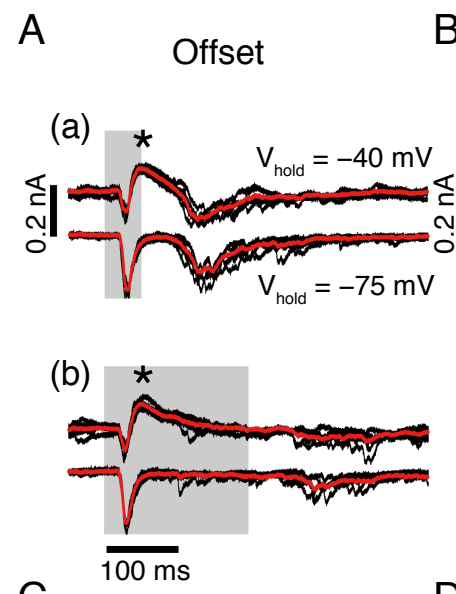

(a)

B Onset-Offset

(a)

(b)

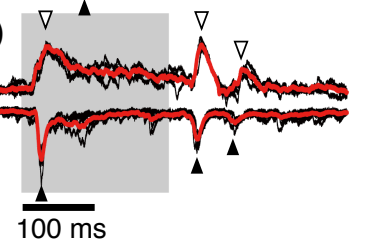

D (a)

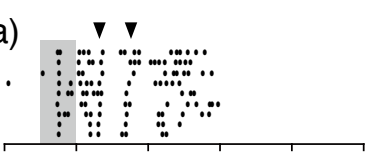

(b)

(b)
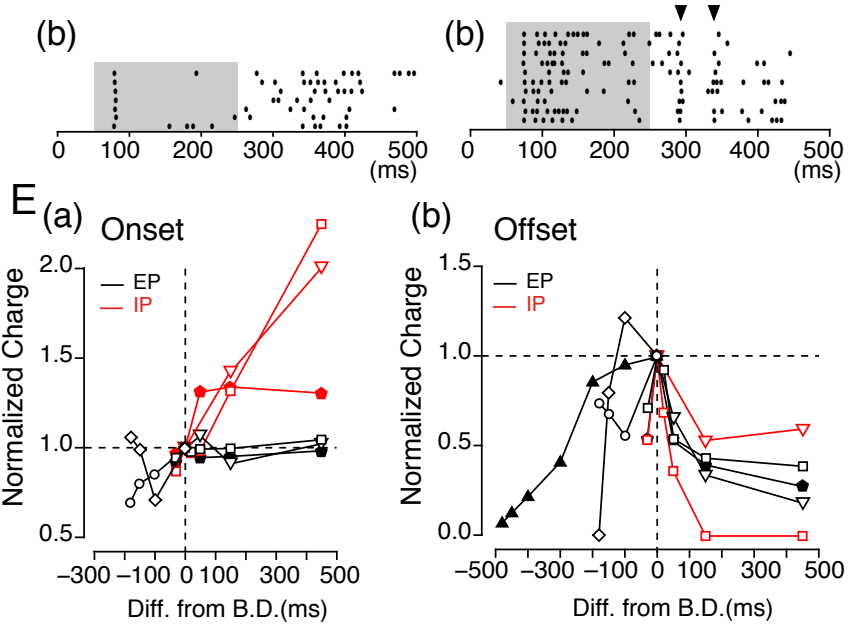

(b) Offset

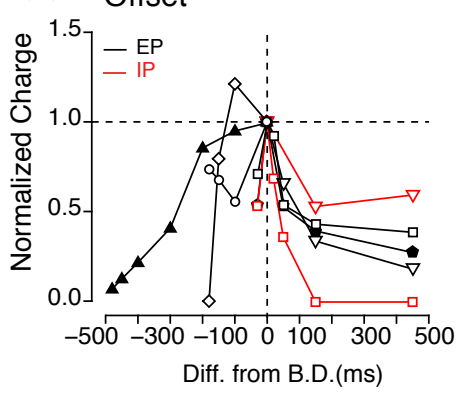


Figure 6
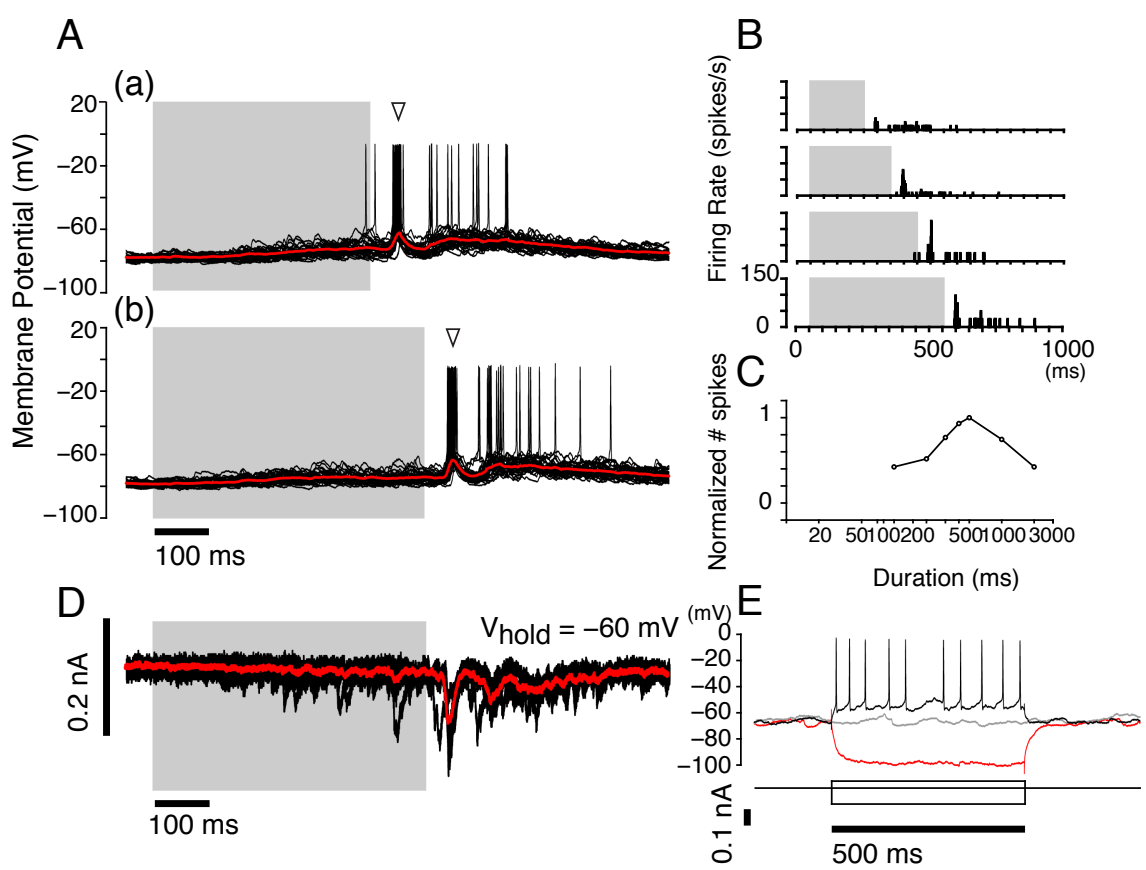
Figure 7
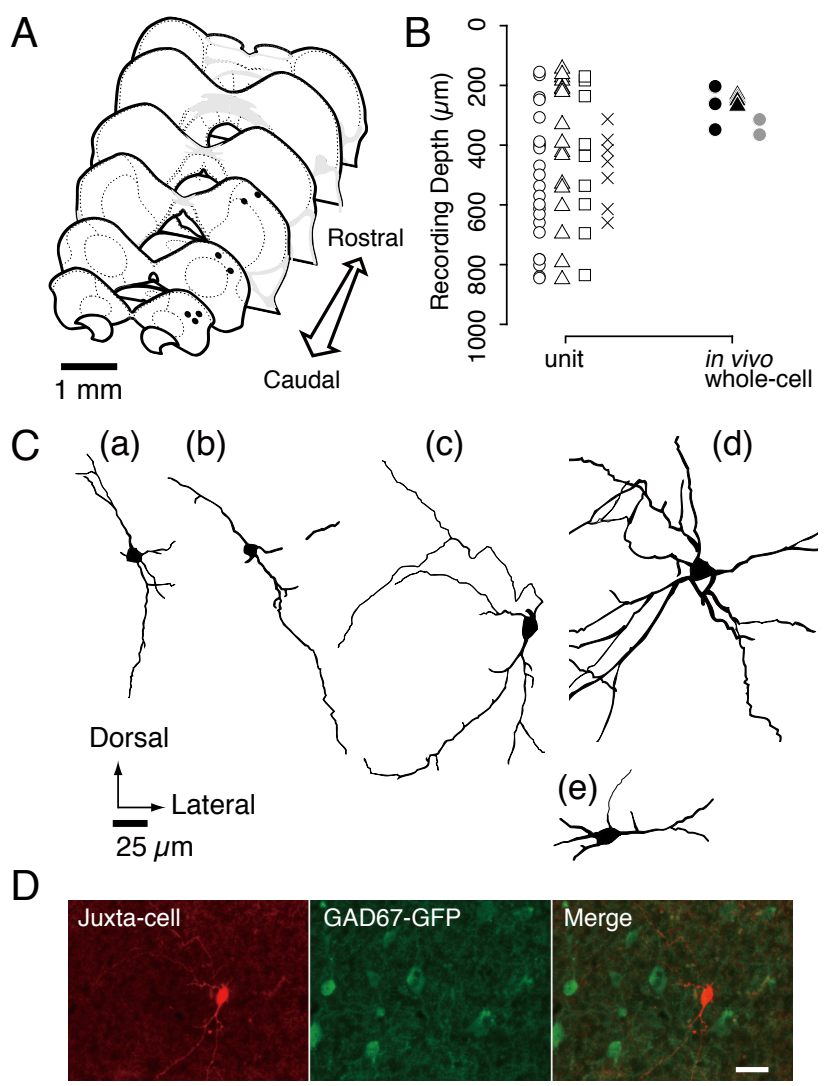